\title{
Transverse Colonic Volvulus in a Child: Successful Management with Decompression and Delayed Laparoscopic Colopexy
}

\author{
Caroline Smith ${ }^{1}$ Alexander Cho ${ }^{1}$ Thomas Tsang ${ }^{1}$ \\ ${ }^{1}$ Department of Paediatric Surgery, Norfolk and Norwich University \\ Hospital, Norwich, United Kingdom \\ Eur J Pediatr Surg Rep 2013;1:48-50.
}

\begin{abstract}
Address for correspondence Caroline Smith, MBChB, MSc, MRCS, Department of Paediatric Surgery, Norfolk and Norwich University Hospital, Jenny Lind Children's Hospital, Colney Lane, Norwich NR4 7UY, United Kingdom (e-mail: c.m.smith@doctors.org.uk).
\end{abstract}

\author{
Abstract \\ Keywords \\ - large bowel volvulus \\ - minimal access \\ surgery \\ - transverse colon
}

We report a case of colonic volvulus in a 7-year-old child with normal development. Operative findings at laparotomy showed a 270-degree transverse colonic volvulus (TCV), with colonic ligamentous attachment abnormalities. A loop ileostomy was formed following detorsion, with delayed laparoscopic colopexy. This is the first case to describe decompression by diversion ileostomy as acute management of TCV with delayed laparoscopic colopexy, so avoiding unnecessary bowel resection.

\section{Introduction}

Transverse colonic volvulus (TCV) in children is a rare condition and as such there is little evidence as to the management in both an acute episode and the long term. The literature consists of case reports and a few case series with two or three patients, with no comparison of management techniques. Previous papers ${ }^{1-3}$ have recommended resection of the colon either acutely or delayed depending on the viability of bowel at the presenting episode. We present a case of TCV managed with acute decompression with a loop ileostomy and interval colonic fixation to prevent recurrence.

\section{Case Report}

A 7-year-old boy, with no history of developmental delay, presented with abdominal pain and vomiting, and a history of no spontaneous passage of stool for 5 days. He was afebrile, with a heart rate of 118 beats per minute but was otherwise hemodynamically stable. His abdomen was distended with palpable loops of bowel. He had generalized tenderness on deep palpation but no peritonism. Bowel sounds were high pitched. Digital rectal examination revealed a rectum loaded with hard feces. A plain abdominal radiograph showed an abnormally dilated transverse colon, but no sequential dilation above, and feces in the rectum ( $\sim$ Fig. 1). Bloods tests revealed a normal count with no leukocytosis and normal CRP.

The initial impression was of fecal impaction and a phosphate enema was given. Response to the enema was poor. Subsequently, the abdominal pain worsened and examination revealed localized upper abdominal tenderness. Repeat abdominal radiograph revealed progressive colonic dilatation with suspected volvulus. The child was taken to the operating theater. Initial colonoscopy showed soft stool in rectum, empty sigmoid, and descending colon; and colonoscope was unable to advance beyond the splenic flexure. A midline laparotomy was performed where a massively dilated, twisted transverse colon was found. A 270-degree clockwise volvulus was reduced and inspection revealed no obvious gangrenous bowel ( - Fig. 2). Normal small bowel rotation but an absent hepatocolic and abnormal splenocolic ligaments were noted. Following detorsion, a diversion loop ileostomy was formed.

Postoperative course was uneventful with stoma becoming active after 24 hours. Biopsies of rectum and proximal transverse colon taken during laparotomy showed normal ganglion cells. The distal loopogram performed 2 months post detorsion suggested the colon had returned to normal caliber and peristalsis was seen. He was started on weekly distal stoma washouts and was brought back 4 months later for received

December 26, 2012

accepted after revision

March 15, 2013

published online

May 9, 2013 (c) 2013 Georg Thieme Verlag KG Stuttgart · New York
DOI http://dx.doi.org/ 10.1055/s-0033-1345102. ISSN 2194-7619. 


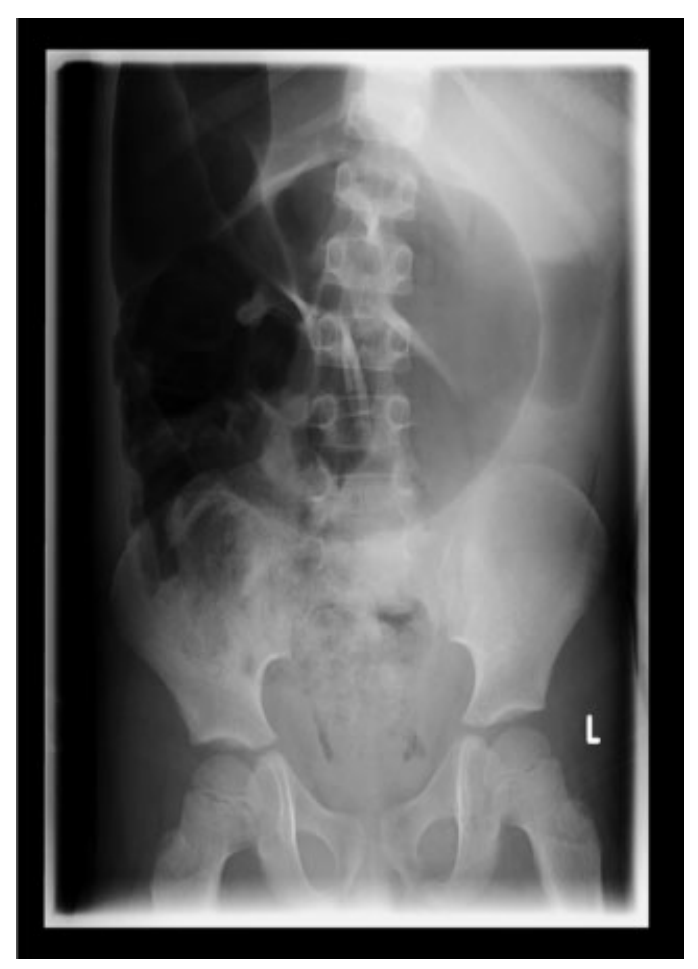

Fig. 1 Abdominal radiograph showing colonic dilatation, feces in the rectum and no proximal small bowel dilatation.

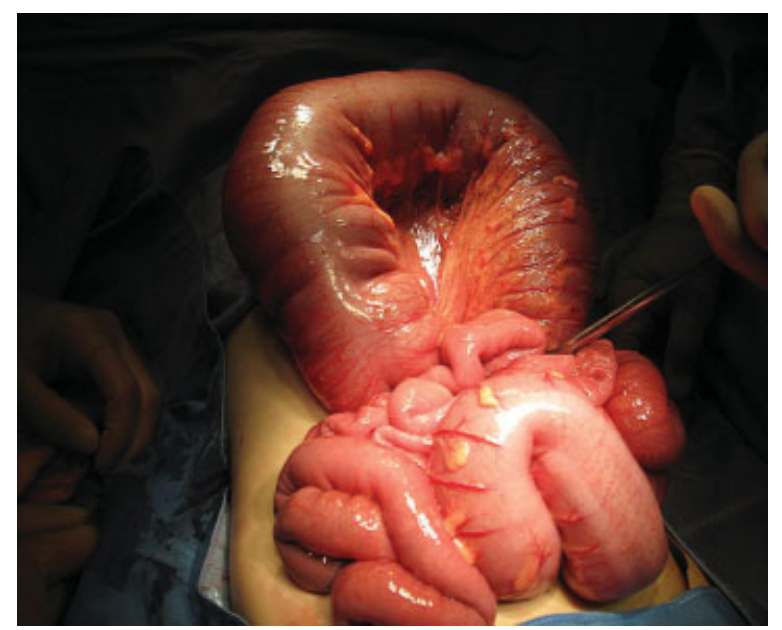

Fig. 2 Image showing the transverse colon following detorsion of the 270-degree volvulus.

laparoscopic-assisted stoma closure. The colon was found to be mildly dilated with some redundant length at this time. A decision was made to fix the transverse colon rather resect the viable colon. The midpoint of the transverse colon was fixed at multiple points to the ligamentum falciforme, laparoscopically, using nonabsorbable suture, ensuring anatomical positioning of the colon. He has been reviewed in clinic 18 months following closure and is thriving and opening bowels regularly using a small amount of laxative.

\section{Discussion}

TCV in children is a rare entity ${ }^{1}$ and, including our patient, we have found 40 cases reported in the worldwide literature. Large bowel volvulus (LBV) is less common in children than in adults and seems to be particularly rare in children younger than 10 years of age. ${ }^{4}$ Furthermore, TCV represents only $1.5 \%$ of LBV in children. ${ }^{1}$ Classically, TCV presents as other LBV with abdominal pain and vomiting, with a history of chronic constipation. Children may have had previous admissions with similar gastrointestinal complaints and constipation. The literature stresses the increased risk of TCV in children with colonic dysmotility, in particular, with global development delay, cerebral palsy, and myotonic dystrophy. ${ }^{1}$ Our patient has a normal development with no history of any underlying neuromuscular diagnosis.

It has been reported that aberrant fixation from the second stage of fetal gastrointestinal rotation leads to an elongated transverse colon and narrowed mesentery because of abnormal ligamentous fixation of the colon. This can lead to chronic constipation and an increased risk of volvulus. ${ }^{3,5}$ This hypothesis is consistent with the findings in this case and would explain the development of intractable constipation, without underlying dysmotility in this child.

TCV presents with septic shock more commonly than other types of $\mathrm{LBV},{ }^{5}$ and rates of gangrene have been reported between 16 and $60 \%{ }^{2,6}$ Mortality from TCV is estimated between 14 and $29 \%{ }^{4,7}$ The viable transverse colon after detorsion in this patient differs from many of cases reported where acute fulminant bowel necrosis and gangrene is found at laparotomy. In view of the viable bowel after detorsion and lack of any significant previous gastrointestinal history, acute resection was not necessary. A loop ileostomy was brought out as a temporary measure. Resting the bowel by proximal diversion allowed investigation into the underlying causes of dysmotility in this child, in particular, to rule out Hirschsprungs and to monitor the response of the colon with alleviation from fecal loading.

Because the bowel caliber returned to normal postoperatively, as shown on contrast study at 2 months, and biopsies did not show any histological abnormalities, resection of the colon was not felt required as abnormal anatomical fixation could be ameliorated by iatrogenic fixation. Findings at elective stoma closure showed some continuing dilatation and redundancy. Colopexy was performed to correct the abnormal fixation of the transverse colon. Since stoma closure and colopexy, the child had satisfactory progress without showing any change in stooling habit.

Although resection of the redundant colon is often advocated because of high recurrence rates, we believe colopexy can protect against this, avoiding major colonic resection and the associated consequences. In the event of future admission with recurrent volvulus, resection would have to be considered because of the failure of fixation.

This case highlights that while TCV must be considered in neuromuscularly impaired children presenting with abdominal pain, vomiting, and a history of constipation, the diagnosis must not be overlooked in children with normal neuromuscular status. 
50 Transverse Colonic Volvulus in a Child Smith et al.

Furthermore, this is the first case describing formation of loop ileostomy following acute detorsion with delayed laparoscopic colopexy. Classically, the ubiquitous redundant transverse colonic loop is resected either acutely or at an interval to prevent recurrence. Initial decompression, without resection, and delayed colopexy offer a safe, conservative approach in the case of nonnecrotic TCV.

Conflict of Interest

None

\section{References}

1 Asabe K, Ushijima H, Bepu R, Shirakusa T. A case of transverse colon volvulus in a child and a review of the literature in Japan. J Pediatr Surg 2002;37(11):1626-1628
2 Samuel M, Boddy SA, Capps S. Volvulus of the transverse and sigmoid colon. Pediatr Surg Int 2000;16(7):522-524

3 Liolios N, Mouravas V, Kepertis C, Patoulias J. Volvulus of the transverse colon in a child: a case report. Eur J Pediatr Surg 2003; 13(2):140-142

4 Folaranmi SE, Cho A, Tareen F, Morabito A, Rakoczy G, Cserni T. Proximal large bowel volvulus in children: 6 new cases and review of the literature. J Pediatr Surg 2012;47(8):1572-1575

5 Samuel M, Boddy SA, Nicholls E, Capps S. Large bowel volvulus in childhood. Aust N Z J Surg 2000;70(4):258-262

6 Barroso Jornet JM, Balaguer A, Escribano J, Pagone F, Domenech J, del Castillo D. Chilaiditi syndrome associated with transverse colon volvulus: first report in a paediatric patient and review of the literature. Eur J Pediatr Surg 2003;13(6): 425-428

7 Hsueh C-C, Jaw T-S, Lin J-Y, Chan HM. Splenic flexure colonic volvulus: a pediatric case report. Kaohsiung J Med Sci 2007;23 (4):207-210 